\title{
Full MAC System Demonstration of Extended 10G-EPON Uplink with 512 ONU Splits Access Span via Burst-Mode SOA and Enhanced-FEC combined with Burst-Mode 3R
}

\author{
Irene Yang $^{*}$ Naoki Suzuki**, Takeshi Suehiro ${ }^{* *}$, Junichi Nakagawa** and Takashi Mizuochi** \\ Electrical Engineering, California Institute of Technology (Caltech), Pasadena, CA 91126, USA \\ E-mail: iyang@caltech.edu \\ "* Information Technology R\&D center, Mitsubishi Electric Corporation, 5-1-1 Ofuna, Kamakura, Kanagawa, Japan \\ E-mail:Suzuki.Naoki@dy.MitsubishiElectric.co.jp
}

\begin{abstract}
This first Extended 10G-EPON uplink system test achieved the largest access span loss of $37 \mathrm{~dB}$ supporting $512 \mathrm{ONU}$ splits over $25 \mathrm{~km}$ with an enlarged loss budget of $51.2 \mathrm{~dB}$ via wide linear gain region burst-mode SOA, RS $(255,223)$ E-FEC and burst-mode $3 \mathrm{R}$.

OCIS codes: (060.4250) Networks; (060.4510) Optical communications
\end{abstract}

\section{Introduction}

Following 10-Gigabit Ethernet Passive Optical Network (10G-EPON) standardization [1] and related research [2, 3], the world finds it needs even more connectivity over longer distances. Thus the idea of Extended PON, currently being studied at IEEE 802.3 [4], was introduced. Extended PON offers cost-insensitive Fiber-to-the-Home (FTTH) services that expands coverage area from near-end urban users to far-end rural users and increases the number of accommodated subscribers. However, bridging this digital divide is not easy; Extended PON requires large loss budgets not previously achieved by standard 10G-EPON. Particularly, China's 10G-EPON standards [5] focus on using new larger loss budget classes of 10GBASE-PR40 (loss budget $33 \mathrm{~dB}$ ) and -PR50 (loss budget $37 \mathrm{~dB}$ ).

In order to achieve such a high loss budget, techniques like $10 \mathrm{~Gb} / \mathrm{s}$ PON utilizing a Praseodymium-Doped Fiber Amplifier (PDFA) based burst-mode amplifier [6, 7], and 1Gb/s PON using a gain-clamped semiconductor optical amplifier (SOA) [8] have been proposed. These techniques employ idealized Forward Error Correction (FEC) or cannot clarify FEC penalties, though other independent studies have found a large burst-mode FEC error [9]. Moreover, never before had a full-system $37 \mathrm{~dB}$ access span loss Extended 10G-EPON test been tried.

Thus, in this paper, we demonstrate the first end-to-end full Media Access Control (MAC)-based system test of an Extended 10G-EPON uplink with RS $(255,223)$ Enhanced-FEC supported novel wide linear gain region burst-mode SOA combined with a burst-mode continuous threshold-tracking control receiver and a $82.5 \mathrm{GS} / \mathrm{s}$ sampling Clock and Data Recovery (CDR) circuit. With an extended loss budget of $51.2 \mathrm{~dB}$, a $24 \mathrm{~dB}$ dynamic range and a high access span loss of $37 \mathrm{~dB}$ (supporting $512 \mathrm{ONU}$ splits over $25 \mathrm{~km}$ ), superior to previous $10 \mathrm{~Gb} / \mathrm{s}$ Extended PON tests with access span loss $31.5 \mathrm{~dB}$ [6], were achieved.

\section{Extended 10G-EPON Uplink MAC System}

Fig. 1 illustrates the experimental setup of the proposed Extended 10G-EPON uplink end-to-end MAC system. The ONU (optical network unit) houses our burst-mode optical transmitter [10]. The OLT (optical line terminal) receiver consists of a 10G-EPON 10GBASE-PR30 compliant burst-mode optical receiver [2] and CDR [3]. In this experiment, the OLT traditionally located at the access node (at the edge of the access span) is replaced by the burst-mode optical amplifier unit and the OLT is relocated to the central office via the trunk span.

Our XFP-based burst-mode ONU transmitter has the very short burst-mode turn-on/off time of 15 ns [10] and its output wavelength is assigned to $1307.8 \mathrm{~nm}$ to utilize peak gain of the burst-mode optical amplifier unit. The output power and the extinction ratio are $+7.0 \mathrm{dBm}$ and $7.1 \mathrm{~dB}$, respectively. The OLT receiver uses our developed

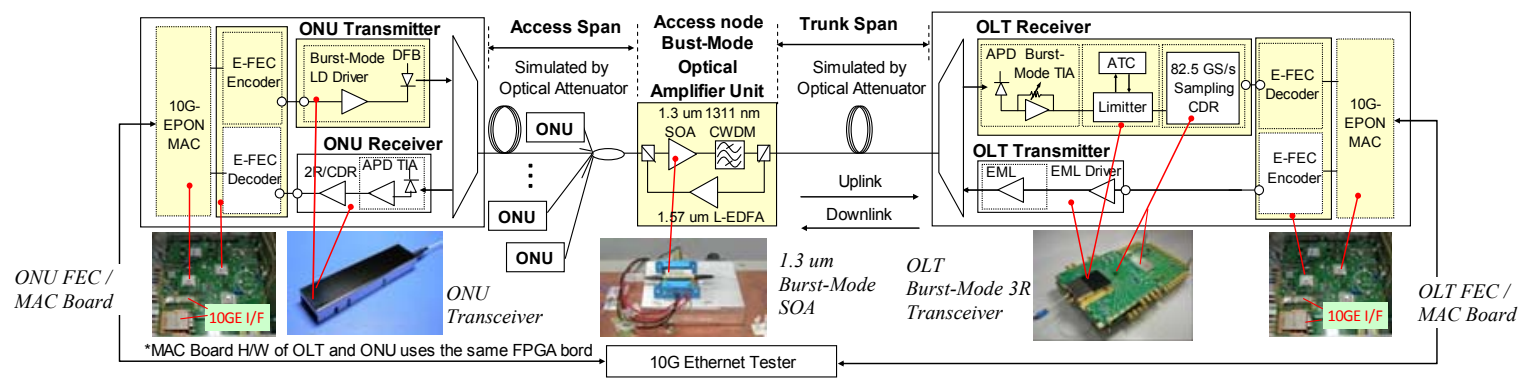

Fig. 1. Experimental setup of the proposed Extended 10G-EPON uplink end-to-end MAC system 
APD-based burst-mode Transimpedance Amplifier (TIA) and limiting amplifier ICs. Its salient features are the fast consecutive TIA automatic gain control (AGC) and automatic threshold control (ATC) which continuously tracks the optimum threshold level of the received burst signal in the limiting amplifier. An $82.5 \mathrm{GS} / \mathrm{s}$ sampling CDR is used for the burst-mode CDR. This burst-mode 3R offers a high-speed burst-mode sync-time of 800 ns [2]. Our Extended PON system's centerpiece is the burst-mode optical amplifier unit: we employ a 1.3 um SOA with a large optical confinement MQW structure and a fast transient gain response of less than $1 \mathrm{~ns}$. A standard $1311 \mathrm{~nm}$ CWDM optical filter is placed at the SOA's output to handle wavelength variation of uncooled ONU transmission and reduce the SOA's ASE. MAC circuits which control uplink burst test packet sequences and RS(255, 223) Enhanced-FEC en/de coding circuits are implemented in FPGAs in the OLT and ONU. The downlink is also connected to handshake PON links using a normal 1.57 um L-EDFA. The system loss budgets are dominated by the uplink. The access span (which includes PON splitters) and trunk span loss are simulated using optical attenuators.

\section{Performance Results}

Fig. 2 shows static gain characteristics of the $1.31 \mathrm{um}$ SOA measured at $1307.8 \mathrm{~nm}$ with an injection current of 270 mA. Fig. 2 (a) demonstrates the gain spectrum of the optical amplifier unit at the output if the 16 nm bandwidth CWDM centered around $1310 \mathrm{~nm}$. Fig. 2 (b) shows the SOA's wide linear-gain region for input powers less than $-15 \mathrm{dBm}$; here, average linear gain is a high $15.7 \mathrm{~dB}$ and the average noise figure (NF) is $9.7 \mathrm{~dB}$. Furthermore, Fig. 3 shows the transient waveforms at the input and output of the SOA and output of the burst-mode optical receiver for a test burst packet of $-22 \mathrm{dBm}$ inserted immediately after a high-level dummy packet of $-6 \mathrm{dBm}$. As the ONU transmitter power is set to $+7 \mathrm{dBm}$, these test burst packets correspond to an access span loss of $29 \mathrm{~dB}$. Most importantly, Fig. 3 ascertains the optical amplifier unit's ability to fully recover burst-mode data without transient waveform distortions within the $800 \mathrm{~ns}$ sync pattern time.

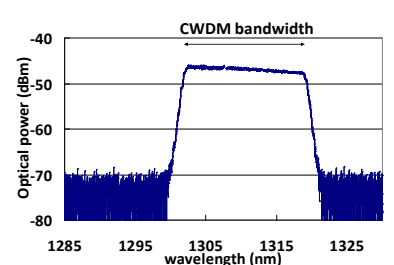

(a) Gain spectrum (CWDM output)

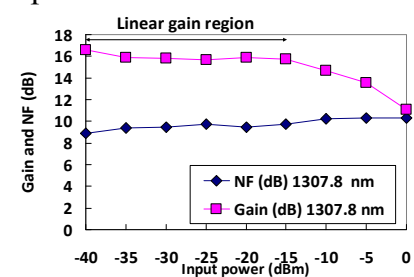

(b) Gain and noise figure graph

Fig. 2. Static characteristics of the burst-mode optical amplifier unit

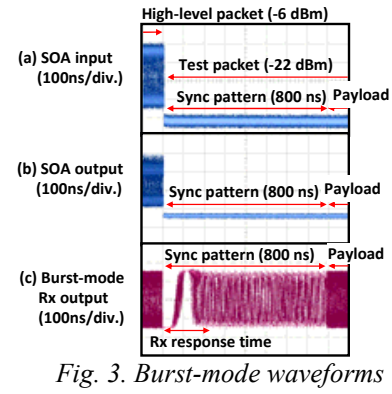

Fig. 3. Burst-mode waveforms

Fig. 4 shows the measured results of burst-mode BER tests of the Extended 10G-EPON uplink system when access span loss was varied at (a) $13 \mathrm{~dB}$, (b) $29 \mathrm{~dB}$, (c) $33 \mathrm{~dB}$ and (d) $37 \mathrm{~dB}$; the optical spectrums at the OLT receiver inputs are also shown as insets. $13 \mathrm{~dB}$ access span loss represents the 10G-EPON standard maximum received power at the access node for $-6 \mathrm{dBm}$ input to the $\mathrm{SOA}$ and $37 \mathrm{~dB}$ corresponds to the largest loss budget class of 10GBASE-PR50. In the test, the upstream burst test packets have 10G-EPON standard specified sync times of $512 \mathrm{~ns}$ for ONU transmitter turn-on/off time and $1200 \mathrm{~ns}$ for OLT receiver settling time [1]. The burst delimiter, payload data, and an end of burst delimiter comprise the rest of the burst packet. The payload consists of sections of 223 data bits followed by 32 parity bits necessary for $\operatorname{RS}(255,223)$ FEC decoding. Burst packets are separated by about 2 us of guard time (allocated by the MAC circuits). The transmitted Ethernet MAC frame size was 64 bytes. Furthermore, as only the frame error rate (FER) was directly measurable by the Ethernet frame tester, the BER was calculated from the FER via the formula below (where $\mathrm{n}$ is the number of bits per Ethernet MAC frame):

$$
B E R=1-(1-F E R)^{1 / n}
$$

At $\mathrm{BER}=10^{-12}$ with a $24 \mathrm{~dB}$ wide input dynamic range of the $\mathrm{SOA}$, the minimum post-FEC burst-mode received sensitivities at access span losses $13 \mathrm{~dB}, 29 \mathrm{~dB}, 33 \mathrm{~dB}$, and $37 \mathrm{~dB}$ were $-27.4 \mathrm{dBm},-28.5 \mathrm{dBm},-27.1 \mathrm{dBm}$, and $-23.3 \mathrm{dBm}$, respectively (represented as the solid blue arrow pointing down in the figures). At powers higher than these, error-free operation was observed. Here, any SOA-induced power penalties were mainly caused by OSNR degradation by output ASE of the SOA. However it should be noted that burst-mode penalties due to the SOA and our $3 \mathrm{R}$ were less than $0.4 \mathrm{~dB}$ at BER $10^{-12}$ post FEC. Further, the burst-mode FEC penalty (i.e. the power penalty from receiver sensitivity at $\mathrm{BER}=10^{-3}$ without $\mathrm{FEC}$ ) were less than $0.15 \mathrm{~dB}$ for access span losses between $29 \mathrm{~dB}$ and $37 \mathrm{~dB}$. In contrast with previous studies done on burst-mode FEC operation, our results indicate a much lower FEC power penalty: this is because our novel burst-mode optical receiver continuously tracks the averaged power of received burst signals and keeps the threshold level optimum [9]. The penalty was rather severe in the case of $13 \mathrm{~dB}$ access span loss as the input power was in the deep saturation region of the SOA due to its chirp induced penalty. 


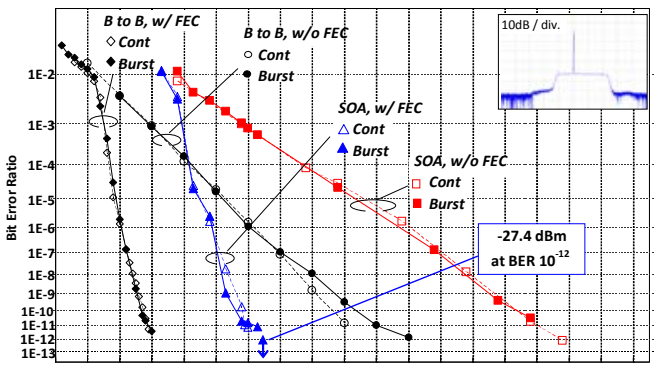

$\begin{array}{lllllllllllllllllll}-34 & -33 & -32 & -31 & -30 & -29 & -28 & -27 & -26 & -25 & -24 & -23 & -22 & -21 & -20 & -19 & -18 & -17 & -16\end{array}$ (a) $13 \mathrm{~dB}$ access span loss

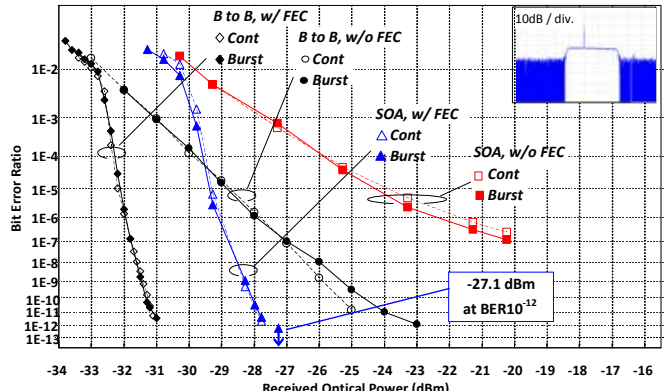

(c) $33 \mathrm{~dB}$ access span loss

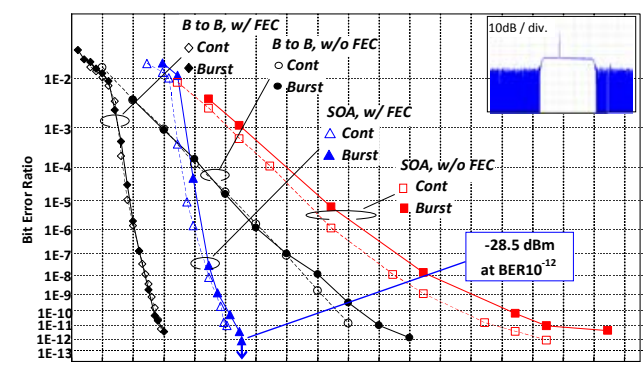

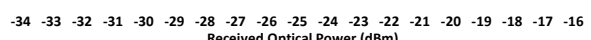
(b) $29 \mathrm{~dB}$ access span loss

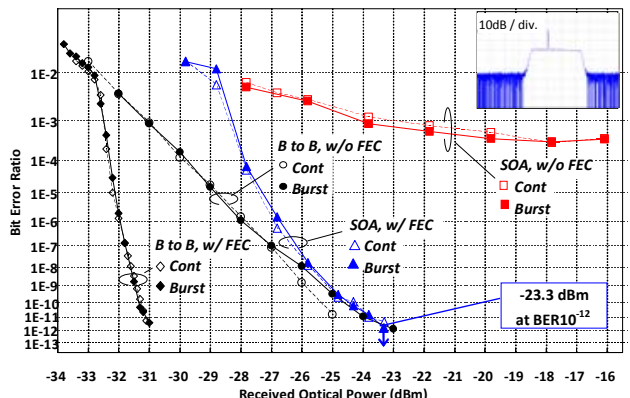

(d) $37 \mathrm{~dB}$ access span loss

Fig. 4. Burst-mode BER curves for our Extended 10G-EPON uplink system at varying access span loss values

Fig. 5 shows measured results for trunk span loss (calculated using ITU-T G.652 optical fiber loss of 0.44 $\mathrm{dB} / \mathrm{km}$ at $1270 \mathrm{~nm}$ ) versus access span loss at BER $=10^{-12}$ with FEC in burst-mode. Under the standard 10G-EPON PR30 uplink with access span loss $29 \mathrm{~dB}$, trunk span loss was successfully increased to $22.2 \mathrm{~dB}$, achieving a total loss budget of $51.2 \mathrm{~dB}$ (see Fig.5 (a)). Moreover, access span loss can be enlarged to $37 \mathrm{~dB}$ with a trunk span loss of $9 \mathrm{~dB}$ (with a high $46.1 \mathrm{~dB}$ total loss budget). Using these obtained budgets, total available span for a given number of splits was calculated in Fig. 5 (b). Assuming standard worst case splitter loss [1], 128 ONU splits over $54.5 \mathrm{~km}$ at an access span loss of $29 \mathrm{~dB}$, and 512 splits over a $25 \mathrm{~km}$ total span distance at the access span loss of $37 \mathrm{~dB}$ were effectively achieved. Compared to previous experiments (which utilized idealized FEC whereas ours did not), our attained $24 \mathrm{~dB}$ dynamic range was $1.5 \mathrm{~dB}$ higher, and our $37 \mathrm{~dB}$ access span loss was $5.5 \mathrm{~dB}$ larger [6].

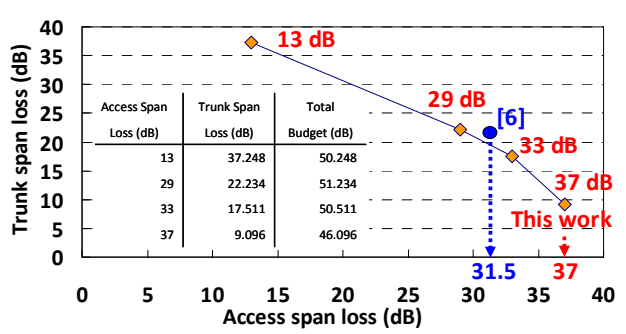

Fig. 5 (a). System Budget results

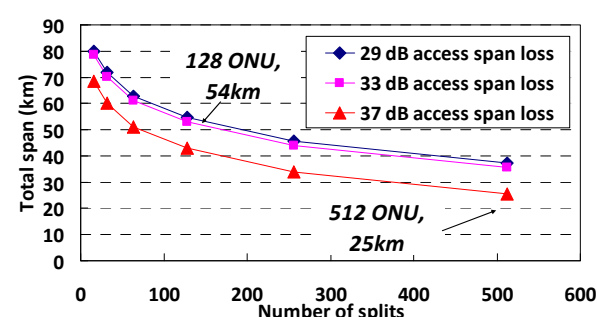

Fig. 5 (b). Total span length available for given number of splits

\section{Conclusions}

We've demonstrated the first full MAC system test of an Extended 10G-EPON uplink using a novel wide linear gain region burst-mode SOA and RS $(255,223)$ Enhanced-FEC combined with burst-mode 3R ICs. With an extended loss budget of $51.2 \mathrm{~dB}$, a large access span loss of $37 \mathrm{~dB}$ (higher by $5.5 \mathrm{~dB}$ than [6]) supporting 512 ONU splits over 25 $\mathrm{km}$, along with an overall access span dynamic range of $24 \mathrm{~dB}$ was achieved. From these results, we've confirmed the ability of our 10G-EPON system to act as an Extended 10G-EPON system to support the global growth of lower user cost next-generation optical access networks.

\section{References}

[1] IEEE Std. 802.3, (2009).

[2] J. Nakagawa et al., OFC/NFOEC2010, PDPD10, (2010).

[3] N. Suzuki at al., OFC/NFOEC2011, OThT3, (2011).

[4] http://www.ieee802.org/3/EXTND_EPON/index.html.

[5] http://www.ccsa.org.cn/english/.
[6] M. Fujiwara et al., ECOC2010, PDP3.2, (2010).

[7] K-I. Suzuki et al., ECOC2011, Mo.1.C.3, (2011)

[8] N.Cheng et al., OFC/NFOEC2011, NTuD7, (2011).

[9] E. In de Betou et al., ECOC2010, Mo.2.B.5, (2010).

[10] E. Igawa et al., OFC/NFOEC2011, NTuD5, (2011). 